\title{
Cost-Effectiveness Analysis of Nintedanib Versus Pirfenidone in Idiopathic Pulmonary Fibrosis in Belgium
}

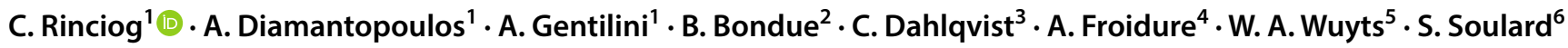

Published online: 14 January 2020

(c) The Author(s) 2020

\begin{abstract}
Background Nintedanib $\left(\mathrm{Ofev}^{\circledR}\right)$ and pirfenidone $\left(\right.$ Esbriet $\left.^{\circledR}\right)$ are recommended by international guidelines as treatment options for idiopathic pulmonary fibrosis (IPF).

Objectives To compare the cost-effectiveness of nintedanib with that of pirfenidone for the treatment of IPF from a Belgian healthcare payer perspective.

Methods The economic analysis used a Markov model that calculated outcomes over patient lifetime. Overall survival was assumed to be the same for the two comparators. Data from a network meta-analysis were used for loss of lung function, acute exacerbation events, safety and treatment discontinuation (for any reason). The health-state utility estimates in the model were calculated from EQ-5D scores collected in nintedanib studies. The assumed resource use for background care was also based on patient-level data that were categorised to fit the health states in the model and synthesised with costs and tariffs from Belgian national databases.

Results Treatment with nintedanib resulted in an estimated total cost of $€ 102,315$, which was less than the total cost of treatment with pirfenidone $(€ 113,313)$. Given the similarities in the survival and progression outcomes obtained with nintedanib and pirfenidone, the model predicted near equivalence in total QALYs (3.353 QALYs for the nintedanib arm and 3.318 for the pirfenidone arm). Results were largely driven by model assumptions underlying mortality, acute exacerbations and treatment discontinuation.

Conclusions After performing a synthesis of the most recently published evidence for IPF patients and assuming a Belgian healthcare payer perspective, we found nintedanib to be more cost-saving than pirfenidone.
\end{abstract}

Electronic supplementary material The online version of this article (https://doi.org/10.1007/s41669-019-00191-w) contains supplementary material, which is available to authorized users.

\section{Rinciog}

crinciog@symmetron.net

1 Symmetron Limited, 8 Devonshire Square, London, UK

2 Department of Respiratory Medicine, Erasme University Hospital, Université Libre de Bruxelles (ULB), Brussels, Belgium

3 Department of Pneumology, CHU UCL Namur Site Godinne, Yvoir, Belgium

4 Department of Pneumology, Cliniques Universitaires Saint-Luc, Université Catholique de Louvain (UCLouvain), Brussels, Belgium

5 Unit for Interstitial Lung Diseases, Department of Respiratory Medicine, University Hospitals Leuven, Louvain, Belgium

6 Boehringer Ingelheim, Amsterdam, The Netherlands

\section{Key Points for Decision Makers}

This analysis provides a synthesis of the most recently published long-term survival evidence for IPF patients and an indirect comparison of other outcomes, including acute exacerbations, loss of lung function, safety and treatment discontinuation (for any reason).

The data were synthesised in an economic model that assumed a Belgian healthcare payer perspective.

Our results indicated that nintedanib and pirfenidone have similar health-related quality of life benefits. Nintedanib had a lower overall cost than pirfenidone, driven by a lower treatment cost and assumptions regarding the risk of acute exacerbations. 


\section{Introduction}

Idiopathic pulmonary fibrosis (IPF) is an incurable chronic lung disease that causes a progressive loss of lung function and is ultimately fatal [1]. Around 11.5 people per 100,000 have IPF in Europe, with an annual incidence of 3.8 new cases per 100,000 [2], although existing publications show wide ranges in the epidemiology of IPF due to differences in study methodology or patient demographics, such as age and gender [3].

Acute exacerbations occur in roughly $10 \%$ of IPF patients annually [4]. These events are defined as periods of unexplained, dramatic worsening of dyspnoea occurring over a short period (usually up to 30 days) and radiographic evidence of further lung injury on top of the existing lung pathology [5]. Acute exacerbations are life-threatening and typically require hospitalisation. Around half of patients hospitalised for an acute exacerbation do not survive the event [6]. Of those admitted to intensive care for acute exacerbation events (about $50 \%$ of patients), around $80 \%$ may die due to either the event itself (70\%), infection (24\%), alveolar haemorrhage $(2 \%)$ or other reasons $(4 \%)$ [6].

Treatment of IPF focuses on slowing disease progression and maintaining lung function (measured via forced vital capacity, FVC), as well as managing symptoms (e.g. coughing, hypoxia) and providing palliative care. Very few disease-modifying pharmacotherapies with proven effectiveness are available [1]. Pirfenidone $\left(\right.$ Esbriet $\left.^{\circledR}\right)$ and nintedanib $\left(\mathrm{Ofev}^{\circledR}\right)$ received authorisation to be marketed as treatments for IPF from the European Medicines Agency (EMA) in 2011 and 2015, respectively. Both agents are recommended by international guidelines as effective therapeutic options for treating IPF patients [7], and are approved for reimbursement by the Belgian Drug Reimbursement Committee (CRM/CTG) under confidential discounts [8].

Long-term data on patient survival are now available for both pirfenidone and nintedanib. In the case of nintedanib, the patient follow-up period extends to 8 years [9-13]. The objective of this study was to adapt an economic model used in the UK and France [14-16]. Specifically, we incorporated long-term survival data for nintedanib and used local cost inputs to evaluate the cost-utility of nintedanib versus pirfenidone from a Belgian healthcare payer perspective.

\section{Methods}

\subsection{Model Structure and Clinical Evidence for the Two Comparators}

The analysis adopted a Belgian healthcare payer perspective and used a Markov model to estimate the health benefits and associated costs of patients with IPF. Costs and quality-adjusted life years (QALYs) were discounted at the standard annual rates of $3 \%$ and $1.5 \%$, respectively [17], and half-cycle correction was incorporated. The model adopted a patient lifetime horizon and a 3-month model cycle, consistent with the intervals between forced vital capacity (FVC) observations in the clinical trials [10].

The structure of the model was based on a cost-effectiveness analysis of nintedanib for IPF in the UK [14] that was submitted to and assessed by NICE as part of Technology Appraisal (TA) 379 [15].

The health states in the model were based on the FVC percent predicted (FVC\% pred) adjusted for the age, gender and height of the patient. Health-state categories reflecting different levels of loss of lung function were defined in increments of 10 percentage points from $\geq 110$ to $<$ 40. The model also incorporated patient history of acute exacerbation events (Fig. 1).

The cohort entered the model at different levels of FVC\%pred and without a history of acute exacerbations, as observed in the INPULSIS clinical trials (see Online Resource 1 in the Electronic supplementary material, ESM). Only disease progression towards worse FVC\%pred states was possible. Acute exacerbations were associated with temporary and long-term effects. Death could occur at any point in the model from any FVC\% pred health state, but was also assigned to all patients for whom the FVC\% pred had dropped below $40 \%$ [1].

The comparators in the analysis were two pharmacological IPF treatments: nintedanib (150 mg twice a day) and pirfenidone (267 mg three times a day). Best supportive care (BSC) was assumed to follow nintedanib or pirfenidone treatment if patients discontinued treatment for any reason.

Relative treatment efficacy for nintedanib and pirfenidone was informed by the results of a network meta-analysis (NMA) developed in-house (Table 1). This analysis was preferred given the availability of TOMORROW and INPULSIS patient-level data that made our meta-analysis more robust than if we had used other published sources. The NMA methodology is described and its results are provided in the supplementary material of a previous publication [14].

To generate the baseline patient survival for nintedanib, patient-level data were pooled from six clinical trials: the 52-week phase II TOMORROW trial (study 1199.30) [9] and its open-label extension (1199.35) [11], the two parallel 52-week phase III trials INPULSIS 1 and 2 [10] (studies 11.99.32 and 1199.34) and their open-label extension INPULSIS-ON [12, 13] (study 1199.33), and a phase IIIb trial with a placebo-controlled period of at least 6 months followed by an open-label period of up to 12 months (study 1199.187) [18]. A Weibull parametric model was used to extrapolate survival beyond the observed trial 
Fig. 1 Model structure. FVC\% $\%$ red forced vital capacity percent predicted
Table 1 Results of the NMA of disease-modifying agents used in IPF patients [14]

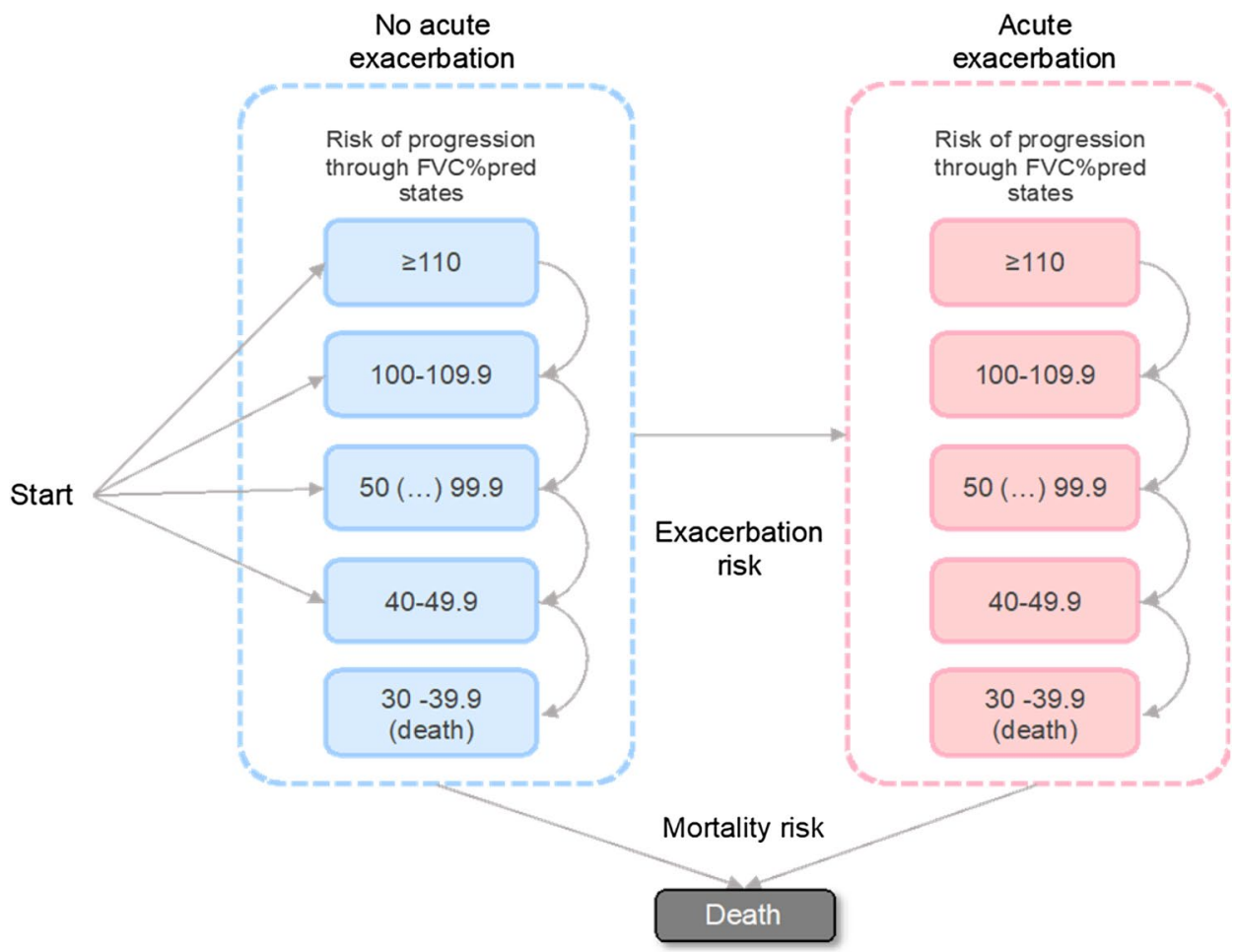

\begin{tabular}{lll}
\hline Outcome & $\begin{array}{l}\text { Nintedanib (300 mg daily) versus } \\
\text { placebo } \\
\text { OR }(95 \% \mathrm{CI})\end{array}$ & $\begin{array}{l}\text { Pirfenidone (2403 mg } \\
\text { daily) versus placebo } \\
\text { OR (95\% CI) }\end{array}$ \\
\hline Overall survival $^{\mathrm{a}}$ & $0.70(0.45-1.09)$ & $0.69(0.45-1.04)$ \\
Acute exacerbations $_{\text {Loss of lung function }}$ & $0.56(0.35-0.89)$ & $1.10(0.43-2.85)$ \\
Serious cardiac events & $0.54(0.42-0.69)$ & $0.55(0.41-0.72)$ \\
Serious gastrointestinal events & $0.76(0.45-1.27)$ & $1.26(0.65-2.49)$ \\
Overall discontinuation & $2.35(1.05-5.88)$ & $0.60(0.23-1.45)$ \\
\hline
\end{tabular}

CI confidence interval, NMA network meta-analysis, $O R$ odds ratio

${ }^{a}$ The OS OR values for nintedanib and pirfenidone vs placebo were used solely in sensitivity analysis scenarios. In the base-case analysis of this cost-effectiveness analysis, we assumed that the survival for patients on pirfenidone was the same as the survival for patients in the nintedanib arm period (Online Resource 2 in the ESM). A comparison of the pooled observed data from the six trials and the Weibull extrapolation is presented in Fig. 2.

The long-term survival data for the two comparators come from open-label extensions of the clinical trials, and a direct or indirect comparison of patient survival was not available. A previous comparison of the randomised controlled trial (RCT) data showed no difference between nintedanib and pirfenidone (odds ratio [OR] 1.02, 95\% CI 0.55-1.87) [14], and other similar NMAs found no statistically significant benefit in OS when the two treatments were considered [19-23]. Therefore, it was assumed that patient survival on pirfenidone was the same as that on nintedanib. This assumption was validated by clinical expert opinion, namely that of co-authors $\mathrm{BB}, \mathrm{CD}, \mathrm{AF}$, and $\mathrm{WW}$, who are practising clinicians with exposure to IPF patients in Belgium. In sensitivity analysis, we replaced the long-term data with the survival data used in the UK model, which was informed by the two nintedanib RCTs, for completeness [9, 10, 24].

Time to first acute exacerbation was based on investigator-reported patient-level data in the INPULSIS trials of nintedanib (Online Resource 2 in the ESM) [10]. Acute exacerbation events were scarce in the INPULSIS trials: 32 out of 426 patients ( $7.5 \%$ ) were observed to experience such an event in the placebo arm, whereas 31 patients out of 640 (4.8\%) experienced such an event in the nintedanib arm during the 52-week trial period. An exponential 


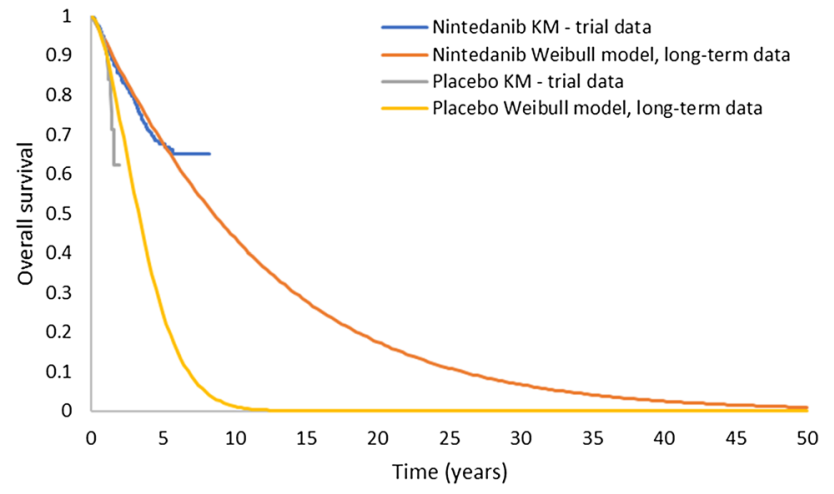

Fig. 2 Comparison of the nintedanib KM trial data and Weibull parametric extrapolation model (please note that the Weibull OS curve presented in the figure is a model input rather than output, and that conclusions regarding patient survival over time should be interpreted with caution). $K M$ Kaplan-Meier

model was fitted to the placebo trial data to estimate the risk over the model lifetime, and a constant risk of $1.97 \%$ was applied each 3-month cycle. The results of our NMA [14] were used to determine the risk of acute exacerbations for nintedanib and pirfenidone (Table 1). Recurrent events were assumed to carry the same risk due to a lack of alternative evidence [14]. Acute exacerbation events were assumed to increase the risk of death by a hazard ratio of 1.4 per cycle post-event [25].

A logistic function was used to estimate patient loss of lung function over time (model A below and Online Resource 2 in the ESM) in the base-case analysis. An alternative function (model B below) that included a covariate whereby patients who had an acute exacerbation would experience accelerated loss in all cycles following such an event was tested in sensitivity analysis.

Model A: $\operatorname{LoLF}_{t 1}=-4.180+0.016 \times \mathrm{FVC} \% \operatorname{pred}_{t 0}$,

Model B: $\operatorname{LoLF}_{t 1}=-4.180+0.016 \times \mathrm{FVC} \% \operatorname{pred}_{t 0}+0.814 \times$ Exa

where $\mathrm{FVC} \%$ pred $_{\mathrm{t} 0}$ is the value of $\mathrm{FVC} \%$ pred at the start of the interval, LoLF is the loss of lung function, and Exa is the exacerbation covariate (indicating whether or not an exacerbation occurred during the previous cycle).

Transitions for patients on nintedanib and pirfenidone were estimated by applying the ORs to the baseline transition defined by the logistic functions (Table 1) [14].

Two serious adverse events (AEs) that were common for both comparators-serious cardiac events and serious gastrointestinal events-were parameterised using OR values from the NMA (Table 1) anchored to placebo baseline risk $(1.39 \%$ per 3 -month cycle for serious cardiac events and $0.42 \%$ per cycle for serious gastrointestinal events [26]). Further AEs of particular clinical interest included gastrointestinal perforation specifically for the nintedanib arm $(0.08 \%$ per cycle) and photosensitivity $(2.32 \%$ per cycle) and rash (6.79\% per cycle) for the pirfenidone arm $[14,24,27]$.

It was assumed that patients on either therapy could discontinue treatment due to any reason. Discontinuation was parameterised using data from our NMA between nintedanib and pirfenidone [14].

\subsection{Health-Related Quality of Life (HRQoL)}

Utility scores for the health states in the model were based on an analysis of patient-level EQ-5D ${ }^{\mathrm{TM}}$ data from the INPULSIS trials [10], with UK preference weights used to derive the utilities [28] (Table 2). Separate analyses of INPULSIS trial data provided estimates for utility decrements associated with acute exacerbations and serious gastrointestinal events [10].

Disutility estimates for other adverse events (i.e. serious cardiac events, gastrointestinal perforation and skin disorders) were obtained from a retrospective analysis of a UK database [14, 29].

\subsection{Cost Inputs}

The model considered all relevant direct healthcare costs, including drug acquisition, treatment-related AEs, monitoring, background follow-up, supplementary oxygen, acute exacerbation and palliative (end-of-life) care costs, in euros. All unit costs were for Belgium $[8,30]$ and were based on 2018 prices.

The list price of nintedanib published in the INAMI database was $€ 2376.21$ per pack ( $€ 7227.64$ cost per 3-month cycle) [30]. The model assumed a daily dose of $300 \mathrm{mg}$ (150 $\mathrm{mg}$ twice daily [10]). The price of pirfenidone was $€ 2472.85$ per pack (€8058.84 cost per 3-month cycle)) [30], assuming a daily dose of $2,403 \mathrm{mg}$ [31] (three $267 \mathrm{mg}$ capsules three times a day). For consistency with the clinical trial data that were used in the model, no dose reduction or escalation was considered in the base-case. Only list prices were used in the analysis, and national or local confidential discounts were not considered. AE-related costs were obtained from the INAMI database using tariffs for each event [30].

Use of nintedanib and pirfenidone was associated with elevated hepatic enzyme values in some patients [31, 32]. Therefore, the base-case model settings assumed one liver panel test every 3 months for all patients. The unit cost for a liver panel test was $€ 48.39[8,30]$.

The background follow-up and acute exacerbation event costs were estimated using patient-level resource use data from the INPULSIS trials and unit costs from Belgium (Table 2 and Online Resource 3 in the ESM) [14]. Patients 
Table 2 HRQoL and cost inputs for the model

\begin{tabular}{|c|c|c|}
\hline Baseline utilities by FVC\%pred status & Mean value (SE) & Source \\
\hline$\geq 110$ & Assumed same value as $90-99.9$ & N/A \\
\hline $100-109.9$ & Assumed same value as $90-99.9$ & N/A \\
\hline $90-99.9$ & $0.8380(0.0083)$ & {$[10,14]$} \\
\hline $80-89.9$ & $0.8105(0.0078)$ & {$[10,14]$} \\
\hline $70-79.9$ & $0.7800(0.0080)$ & {$[10,14]$} \\
\hline $60-69.9$ & $0.7657(0.0084)$ & {$[10,14]$} \\
\hline $50-59.9$ & $0.7387(0.0105)$ & {$[10,14]$} \\
\hline 40-49.9 & $0.6634(0.0258)$ & {$[10,14]$} \\
\hline Acute exacerbation-related disutility & Mean value (SE) & Source \\
\hline Investigator-reported acute exacerbations-first month & $-0.140(0.047)$ & {$[10,14]$} \\
\hline Investigator-reported acute exacerbations-subsequent months & $-0.078(0.032)$ & {$[10,14]$} \\
\hline AE-related disutility & Mean value & Source \\
\hline SC events & -0.0165 & {$[14,29]$} \\
\hline SGI events & -0.0057 & {$[10,14]$} \\
\hline Skin disorders ${ }^{\mathrm{a}}$ & -0.0068 & {$[14,29]$} \\
\hline GI perforation & -0.0098 & {$[14,29]$} \\
\hline Drug acquisition cost (per day) & Mean value & Source \\
\hline Nintedanib & $€ 79.21$ & {$[8,30]$} \\
\hline Pirfenidone & $€ 88.32$ & {$[8,30]$} \\
\hline AE-related costs (per event) & Mean value & Source \\
\hline SC events & $€ 4557.61$ & {$[8,30]$} \\
\hline SGI events & $€ 7031.34$ & {$[8,30]$} \\
\hline Skin disorders & $€ 21.27$ & {$[8,30]$} \\
\hline GI perforation & $€ 21.27$ & {$[8,30]$} \\
\hline Background follow-up costs by FVC\%pred status (per cycle) & Mean value & Source \\
\hline$\geq 110$ & $€ 301.28$ & {$[8,30]$} \\
\hline $100-109.9$ & $€ 271.84$ & {$[8,30]$} \\
\hline $90-99.9$ & $€ 320.04$ & {$[8,30]$} \\
\hline $80-89.9$ & $€ 268.35$ & {$[8,30]$} \\
\hline $70-79.9$ & $€ 325.91$ & {$[8,30]$} \\
\hline $60-69.9$ & $€ 296.86$ & {$[8,30]$} \\
\hline $50-59.9$ & $€ 447.26$ & {$[8,30]$} \\
\hline 40-49.9 & $€ 980.50$ & {$[8,30]$} \\
\hline \multirow[t]{2}{*}{ Acute exacerbation-related cost (per cycle) } & Mean value & Source \\
\hline & $€ 6649.50$ & {$[8,30]$} \\
\hline Other costs (per cycle) & Mean value & Source \\
\hline Patient monitoring (liver panel tests) & $€ 48.39$ & {$[8,30]$} \\
\hline Oxygen supplementation & $€ 775.82$ & {$[8,30]$} \\
\hline Palliative care (EoL) & $€ 3783.61$ & {$[33]$} \\
\hline
\end{tabular}

$A E$ adverse event, EoL end of life, FVC\% pred forced vital capacity percent predicted, GI gastrointestinal, HRQoL health-related quality of life, $N / A$ not applicable, $S C$ serious cardiac, $S E$ standard error, $S G I$ serious gastrointestinal

${ }^{a}$ Rash and photosensitivity reaction were grouped as "skin disorders" 
in health states with FVC\%pred $<80 \%$ were assumed to require supplementary oxygen. The cost of oxygen therapy in Belgium was estimated at $€ 775.82$ per 3-month cycle $[8,30]$. End-of-life palliative care costs of $€ 3783.61$ were applied for the last 30 days of life of each patient [33].

\subsection{Analyses}

Results for the cost-effectiveness of nintedanib versus pirfenidone were presented as an incremental cost-effectiveness ratio (ICER) that considered QALYs and costs accumulated over the cohort lifetime. Despite the fact there is no official cost-effectiveness threshold in Belgium, a willingness-to-pay (WTP) threshold of $€ 30,000$ per QALY gained was assumed, as in previous studies $[34,35]$. Deterministic one-way sensitivity analyses of model parameters, including transition probabilities, costs, utilities and adverse event assumptions, were performed. Additional scenarios focusing on probabilities, safety, FVC\%pred, and several cost assumptions were studied in separate analyses. Probabilistic sensitivity analysis was also conducted (1000 samples) with appropriate distributions fitted to the model variables (Online Resource 4 in

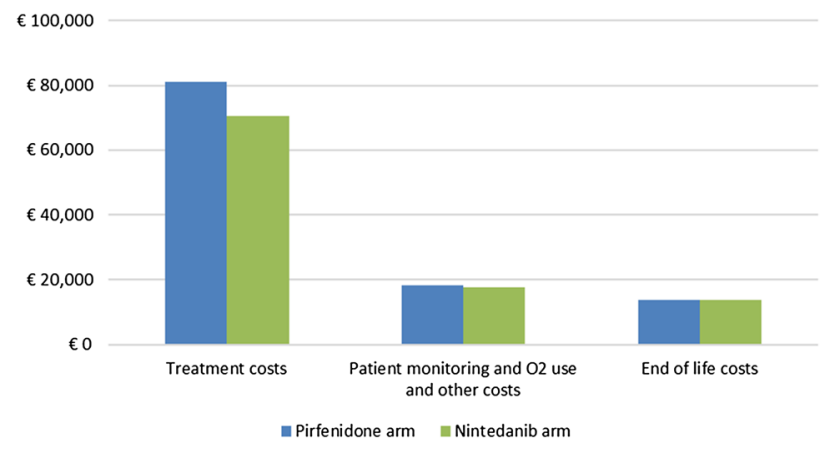

Fig. 3 Total cost comparison for nintedanib and pirfenidone. *Other costs related to acute exacerbations, AEs and liver panel tests the ESM). Additional tests of model convergence were performed to ensure that 1000 iterations were sufficient to minimise the Monte Carlo error (Online Resource 5 in the ESM).

\section{Results}

\subsection{Base-Case Analysis}

The base-case deterministic analysis found that, over the lifetime of a patient, nintedanib treatment accrued fewer overall costs than pirfenidone treatment $(€ 102,315$ versus $€ 113,313)$.

Drug acquisition costs accounted for $69 \%$ of the total cost in the nintedanib arm and $72 \%$ of the total cost in the pirfenidone arm. Other healthcare costs made up $17 \%$ of the total cost for nintedanib and $16 \%$ of the total cost for pirfenidone (Fig. 3). End-of-life palliative care costs were virtually identical in the two arms given that there were no patients alive at the end of the modelled time horizon. The cost savings were driven by the lower list price of nintedanib in Belgium and the lower risk of acute exacerbations.

Given the similarities in the survival and progression outcomes between nintedanib and pirfenidone, the model predicted near equivalence in total QALYs (3.353 QALYs for the nintedanib arm and 3.318 for the pirfenidone arm; Table 3).

\subsection{Deterministic and Probabilistic Sensitivity Analysis}

Model sensitivity was tested by varying each parameter and recording the resulting ICERs for all the main model parameters (see Online Resource 5 of the ESM). The model was sensitive to changes in survival, risk of acute exacerbations and treatment discontinuation. When the lower bounds of the $95 \%$ confidence intervals for survival risk and acute

Table 3 Base-case cost-effectiveness results for nintedanib versus pirfenidone

\begin{tabular}{llll}
\hline & Nintedanib & Pirfenidone & Difference \\
\hline Deterministic analysis & & & $-€ 10,998$ \\
Total costs & $€ 102,315$ & $€ 113,313$ & 0.035 \\
QALYs & 3.353 & 3.318 & 0.004 \\
LYs & 4.371 & 4.367 & -0.104 \\
Exacerbation events & 0.242 & 0.346 & $-€ 10,062(-€ 12,294$ to $-€ 9014)$ \\
Probabilistic sensitivity analysis (average $95 \% \mathrm{CI})$ & & $0.085(0.085-0.105)$ \\
Total costs & $€ 102,901(€ 86,292$ to $€ 122,301)$ & $€ 112,964(€ 95,306$ to $€ 134,594)$ & $0.062(0.047-0.105)$ \\
QALYs & $3.364(2.695$ to $€ 4.175)$ & $3.279(2.611$ to $€ 4.070)$ & $-0.126(-0.513$ to -0.001$)$ \\
LYs & $4.388(3.491$ to $€ 5.471)$ & $4.327(3.445$ to $€ 5.365)$ & $0.372(0.144$ to $€ 0.907)$ \\
Exacerbation events & $0.247(0.142$ to $€ 0.394)$ &
\end{tabular}

$C I$ confidence interval, $L Y s$ life years, $Q A L Y$ quality-adjusted life year 
exacerbation risk were used (scenarios 1-2 in Table 12 of Online Resource 5 in the ESM), the ICER values increased to $€ 4581,807$ and $€ 361,564$ per QALY gained for nintedanib versus pirfenidone, respectively, with both changes driven by higher total costs and QALYs in the pirfenidone arm relative to the nintedanib arm.

The impact of changes in the treatment efficacy of nintedanib and pirfenidone for OS are further explored in Table 13 of Online Resource 5 (scenarios 15-17 and 19-21) in the ESM. The use of long-term survival data for nintedanib and the assumption of equivalence between the interventions were two of the strongest assumptions of this analysis. To further test these assumptions, scenarios 15-17 explored the impact of using survival data observed in the nintedanib RCTs $[9,10]$ and treatment effect assumptions for pirfenidone and nintedanib as reported in Table 1 [14]. Results of these scenarios were consistent with the current analysis, with nintedanib the dominant intervention. Scenarios 19-20 further considered OR values favouring pirfenidone (less than 1) [20], and resulted in positive ICER values, which were explained by greater total costs and QALYs for the pirfenidone arm than for the nintedanib arm. Scenario 21 considered an OR value favouring nintedanib (higher than 1) [20], and resulted in nintedanib dominating over pirfenidone, as in the base-case setting of this analysis.

When overall discontinuation assumptions were changed (scenarios 40-43), ICER values ranged from $€ 51,207$ to $€ 1,272,914$ per QALY gained for nintedanib versus pirfenidone. These effects were also due to greater total costs and QALYs for the pirfenidone arm compared with the nintedanib arm. For all other scenarios tested, nintedanib remained the dominant strategy in this analysis.

Probabilistic results yielded similar total costs $(€ 102,901$ for nintedanib and $€ 112,964$ for pirfenidone) and QALYs (3.364 for nintedanib and 3.279 for pirfenidone) to those derived in the deterministic analysis, as shown in Table 3. Figure 4 shows that the probabilistic results for nintedanib and pirfenidone overlap in terms of incremental QALYs and costs. While the total cost of the pirfenidone strategy was higher than that of nintedanib, the two were nearly equivalent in terms of health benefits (Table 3). For completeness, a PSA cost-effectiveness acceptability curve is presented in Online Resource 5 of the ESM. The nintedanib intervention had a $99.6 \%$ probability of being more cost-effective than pirfenidone at a threshold of $€ 50,000$ per QALY gained.

\section{Discussion}

This was an evaluation of the cost-effectiveness of two relatively new interventions in the treatment of IPF. The analysis showed small differences in costs and QALYs between the two comparators. It was estimated that nintedanib was less costly than pirfenidone due to its lower price and the fact that patients experienced fewer acute exacerbations.

Further analysis showed that, in the current setup, the results were sensitive to changes in the risk of acute exacerbation events, such that nintedanib moved from being a
Fig. 4 Cost-effectiveness scatter plot. $Q A L Y s$ quality-adjusted life years

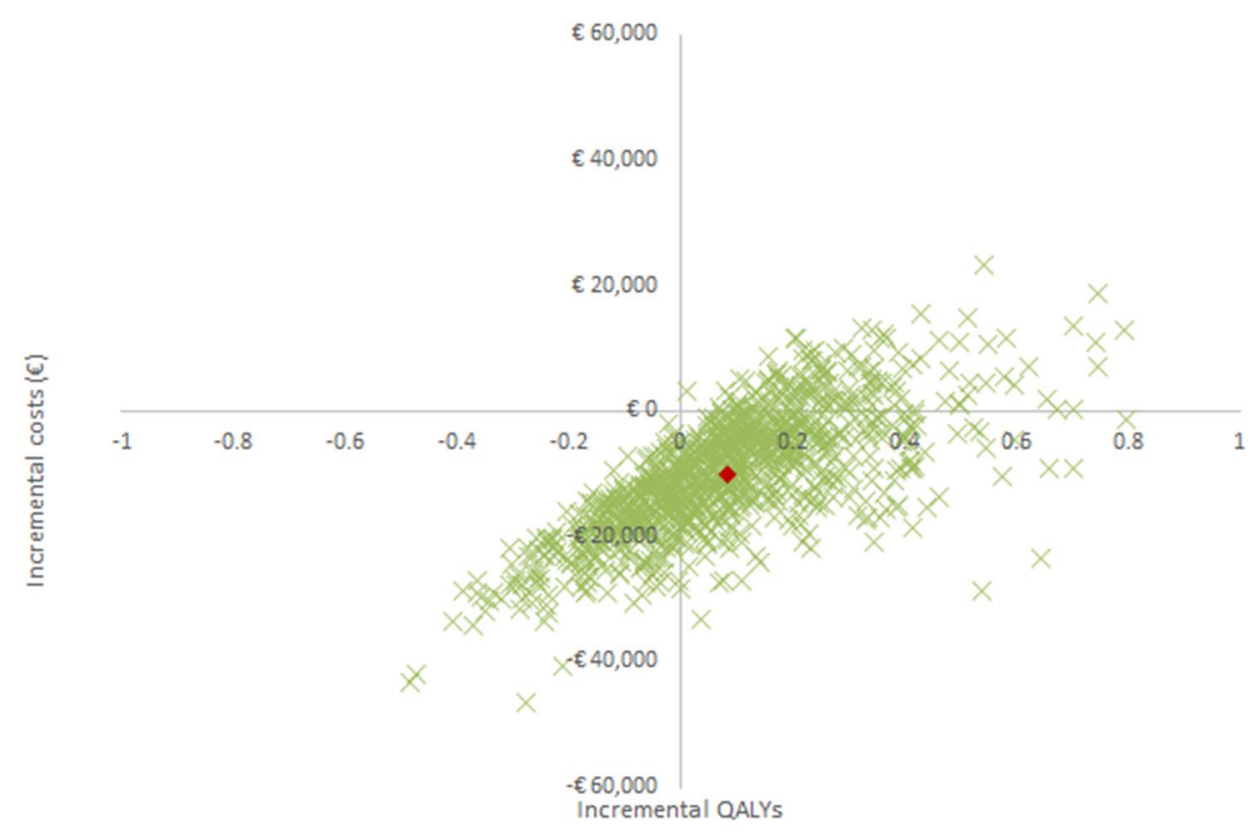


cost-saving treatment to having a positive ICER value above $€ 300,000$ per QALY gained versus pirfenidone. The importance of acute exacerbation events in the economic model is consistent with clinical experience, which focuses on avoiding such debilitating events. Acute exacerbations worsen the advance of the disease considerably, they are costly to manage, and they have strongly negative impacts on the quality of life and subsequent treatment costs for patients who survive such events [4-6].

Our sensitivity analysis scenarios also indicated that the model was sensitive to variations in survival, with greater costs and QALYs observed for the pirfenidone arm than for the nintedanib arm, resulting in highly positive ICER values when using the lower bounds of the $95 \%$ confidence interval for the survival of the nintedanib arm. Similarly, changes in the OS treatment efficacy in favour of pirfenidone generated positive ICER values that were attributable to greater total costs and QALYs in the pirfenidone arm than in the nintedanib arm.

The other driver of cost savings for nintedanib was the drug price. This analysis used the list prices of nintedanib and pirfenidone; no discount was applied. Lower price assumptions for pirfenidone may affect the overall costeffectiveness results and could reduce or eliminate any cost savings. We have estimated that when all other model assumptions and conditions remain the same, the list price of nintedanib will require a $13.5 \%$ increase to be cost neutral relative to pirfenidone.

The conceptualisation of the economic model followed the KCE recommendations for healthcare economic evaluations and international guidelines for best practice in economic modelling $[17,36]$. The health-state utilities in the model were based on patient-level EQ-5D data for IPF patients. The assumed resource use for background care was also based on patient-level data; these data were categorised to fit the model health states and synthesised with costs and tariffs from Belgian national databases.

Previous analyses used clinical evidence from pivotal RCTs to define patient survival $[14,16]$. In a previously published cost-effectiveness analysis assessing nintedanib for the treatment of IPF in the UK, parametric survival models were fitted to 52 weeks of observed data from the pooled TOMORROW and INPULSIS clinical trials, and the best-fit model was used for extrapolation [14]. The present study benefitted from a longer period of observation (the maximum exposure to nintedanib was 93.1 months) and more recorded deaths $(22.5 \%)$ [18] than in the UK analysis (all-cause mortality in the nintedanib arm was $8.24 \%$ in the TOMORROW trial and $5.49 \%$ in the INPULSIS trials) [9, $10]$.

Since the new survival analysis included data from the open-label period of the study [18], only the nintedanib arm was used for extrapolation based on the observed survival of patients who continued with nintedanib from the randomised period. The OS model projection using the Weibull distribution for the nintedanib arm at the end of one year was validated against long-term clinical trial observations [18], as well as against previous extrapolations of RCT OS evidence [9, 10, 14], as shown in Online Resource 6 of the ESM.

As there was no comparison of long-term evidence between nintedanib and pirfenidone at the time we developed the model, it was assumed that there was no difference between the two comparators in patient survival, and the nintedanib-generated survival curve was also used for the pirfenidone arm of the model [9-13]. We note that when the two pivotal RCTs for nintedanib and pirfenidone were previously compared in an NMA, the analysis found no difference between the two comparators [14].

Other relative efficacy estimates were based on a NMA conducted after a systematic review of the literature. The results of this NMA were found to be consistent with those of five other studies [19-23, 37] and were presented in a reimbursement submission in the UK, where NICE gave a positive recommendation of nintedanib for the treatment of IPF in England and Wales [15].

Another potential limitation of the analysis lies in the lack of Belgian-specific utility values. This study used EQ-5D values for FVC\%pred-modelled health states that originated from the overall population of the INPULSIS clinical trials. Further, AE-related utility decrements were based on a study focusing on a UK population [29], given the lack of Belgian-specific published data. Nonetheless, Belgian clinical experts (the co-authors WW, AF, BB, CD) have reviewed the EQ-5D values and utility decrements used in the study and confirmed their applicability for the Belgian population.

A further limitation of the model stems from the rarity of acute exacerbation events in the trials considered. This did not allow a statistically robust analysis of their effect on mortality and disease progression. Consequently, the impact of acute exacerbations may have been underestimated in the present analysis. While we have attempted to estimate the impact of acute exacerbations on disease progression (see Table 8 in Online Resource 2 of the ESM), the associated covariate was not statistically significant ( $p$ value 0.445 ), and was therefore not used in the base-case setting of this analysis. Whilst studies show that patients who experience acute exacerbation events undergo a decrease in quality of life and a considerable increase in healthcare resource use $[3,38]$, further research and long-term data on acute exacerbation events would be required to assess their impact on efficacy outcomes such as mortality and disease progression.

Despite the above limitations, to our knowledge, this analysis is the first to use long-term survival data for IPF patients treated with nintedanib. As patients and clinicians become more familiar with these treatments, their effects 
and their side effects, future studies could compare the long-term outcomes of the cost-effectiveness models with real-world data.

\section{Conclusion}

After performing a synthesis of the most recently published evidence for IPF patients, we found that nintedanib and pirfenidone have similar health-related quality of life benefits, confirming the findings of previous analyses. Assuming a Belgian healthcare payer perspective, we found nintedanib treatment to be cost-saving compared to pirfenidone treatment. The results in our analysis were dependent on the price difference between the two agents and on assumptions regarding the risk of acute exacerbations.

Acknowledgements The authors would like to thank Catherine Druez, a former employee of Boehringer Ingelheim Belgium, for facilitating discussions between the Belgian clinical experts. The authors would also like to thank Jana Tillotson for editorial support. The authors were fully responsible for all content and editorial decisions, were involved at all stages of manuscript development and have approved the final version. The cost-effectiveness model used in this study was provided to the journal's peer reviewers for their reference when reviewing the manuscript.

Author Contributions CR, AD and SS conceived the analysis; CR, $\mathrm{AG}$ and $\mathrm{AD}$ conducted the analysis; $\mathrm{SS}$ organised the adaptation of the model inputs to the Belgian setting; WW, AF, BB and CD proposed clinical input changes and validated the model structure and assumptions; AD, CR and AG wrote the introduction, methods and results; AD wrote the discussion section. All authors provided critical feedback on the manuscript and have approved the final version.

Data Availability Statement The data and methods that support the findings of this study are publicly available and referenced in the manuscript. Details of the inputs used to generate the analysis are presented in the ESM. The Excel file of the cost-effectiveness model was shared with the manuscript reviewers upon request.

\section{Compliance with Ethical Standards}

Funding This study was funded by Boehringer Ingelheim Netherlands. Boehringer Ingelheim was involved in the analysis of clinical trial data.

Conflict of interest CR, AG and AD are employed by Symmetron Ltd, which received funding from Boehringer Ingelheim for this project. $\mathrm{SS}$ is an employee of Boehringer Ingelheim. BB received research grants and consultancy and lecture fees from Boehringer Ingelheim and F. Hoffmann-La Roche. AF received consultancy and lecture fees from Boehringer Ingelheim, F. Hoffmann-La Roche, Novartis, GlaxoSmithKline and AstraZeneca. AF received an unrestricted research grant from Boehringer Ingelheim and F. Hoffmann-La Roche. WW reports the receipt of grants paid to his institution from F. Hoffman-La Roche and Boehringer Ingelheim. CD received grants from Boehringer Ingelheim for clinical trial support and for organising symposia and received consultancy fees from Boehringer Ingelheim.
Open Access This article is licensed under a Creative Commons Attribution-NonCommercial 4.0 International License, which permits any non-commercial use, sharing, adaptation, distribution and reproduction in any medium or format, as long as you give appropriate credit to the original author(s) and the source, provide a link to the Creative Commons licence, and indicate if changes were made. The images or other third party material in this article are included in the article's Creative Commons licence, unless indicated otherwise in a credit line to the material. If material is not included in the article's Creative Commons licence and your intended use is not permitted by statutory regulation or exceeds the permitted use, you will need to obtain permission directly from the copyright holder. To view a copy of this licence, visit $\mathrm{http} / / /$ creativecommons.org/licenses/by-nc/4.0/.

\section{References}

1. National Institute for Health and Care Excellence (NICE). Diagnosis and management of suspected idiopathic pulmonary fibrosis. Idiopathic pulmonary fibrosis: full version. London: National Institute for Health and Care Excellence; 2013.

2. Nguengang Wakap S, editor. Prevalence and incidence of rare diseases: Bibliographic data. Orphanet report series. 2019. Available from: https://www.orpha.net/orphacom/cahiers/docs/ GB/Prevalence_of_rare_diseases_by_alphabetical_list.pdf.

3. Diamantopoulos A, Wright E, Vlahopoulou K, Cornic L, Schoof N, Maher TM. The burden of illness of idiopathic pulmonary fibrosis: a comprehensive evidence review. Pharmacoeconomics. 2018;36(7):779-807.

4. Johannson K, Collard HR. Acute exacerbation of idiopathic pulmonary fibrosis: a proposal. Curr Respir Care Rep. 2013;2(4):233.

5. Collard HR, Moore BB, Flaherty KR, Brown KK, Kaner RJ, King TE Jr, et al. Acute exacerbations of idiopathic pulmonary fibrosis. Am J Respir Crit Care Med. 2007;176(7):636-43.

6. Song JW, Hong SB, Lim CM, Koh Y, Kim DS. Acute exacerbation of idiopathic pulmonary fibrosis: incidence, risk factors and outcome. Eur Respir J. 2011;37(2):356.

7. Raghu G, Rochwerg B, Zhang Y, Garcia CA, Azuma A, Behr J, et al. An official ATS/ERS/JRS/ALAT clinical practice guideline: treatment of idiopathic pulmonary fibrosis. An update of the 2011 clinical practice guideline. Am J Respir Crit Care Med. 2015;192(2):e3-19.

8. Boehringer Ingelheim. Rapport scientifique OFEV (data on file). Ingelheim am Rhein: Boehringer Ingelheim; 2018.

9. Richeldi L, Costabel U, Selman M, Kim DS, Hansell DM, Nicholson AG, et al. Efficacy of a tyrosine kinase inhibitor in idiopathic pulmonary fibrosis. N Engl J Med. 2011;365(12):1079-87.

10. Richeldi L, du Bois RM, Raghu G, Azuma A, Brown KK, Costabel U, et al. Efficacy and safety of nintedanib in idiopathic pulmonary fibrosis. N Engl J Med. 2014;370(22):2071-82.

11. Richeldi L, Kreuter M, Selman M, Crestani B, Kirsten A-M, Wuyts WA, et al. Long-term treatment of patients with idiopathic pulmonary fibrosis with nintedanib: results from the TOMORROW trial and its open-label extension. Thorax. 2018;73(6):581.

12. Wuyts WA, Kolb M, Stowasser S, Stansen W, Huggins JT, Raghu G. First data on efficacy and safety of nintedanib in patients with idiopathic pulmonary fibrosis and forced vital capacity of $\leq 50 \%$ of predicted value. Lung. 2016;194(5):739-43.

13. Crestani B, Quaresma M, Kaye M, Stansen W, Stowasser S, Kreuter M. Long-term nintedanib treatment in idiopathic pulmonary fibrosis (IPF): new data from INPULSIS-ON. Eur Respir J. 2017;50(suppl 61):OA3402.

14. Rinciog C, Watkins M, Chang S, Maher TM, LeReun C, Esser D, et al. A cost-effectiveness analysis of nintedanib in 
idiopathic pulmonary fibrosis in the UK. Pharmacoeconomics. 2017;35(4):479-91.

15. National Institute for Health and Care Excellence (NICE). Idiopathic pulmonary fibrosis-nintedanib (ID752). NICE Technology Appraisal Guidance (TA379). 2016. Available from: https:// www.nice.org.uk/guidance/ta379/ifp/chapter/what-has-nice-said.

16. Porte F, Cottin V, Catella L, Luciani L, Le Lay K, Benard S. Health economic evaluation in idiopathic pulmonary fibrosis in France. Curr Med Res Opin. 2018;34(10):1731.

17. Le Centre Fédéral d'Expertise des Soins de Santé (KCE). Recommandations Belges pour les évaluations économiques et les analyses d'impact budgétaire. Contract no.: KCE report 183B. Brussels: KCE; 2012.

18. Lancaster L, Crestani B, Hernandez P, Inoue Y, Wachtlin D, Loaiza L, et al. Safety and survival data in patients with idiopathic pulmonary fibrosis treated with nintedanib: pooled data from six clinical trials. BMJ Open Respir Res. 2019:6(1).

19. Canestaro WJ, Edwards TC, Patrick DL. Systematic review: patient-reported outcome measures in coeliac disease for regulatory submissions. Aliment Pharmacol Ther. 2016;44(4):313-31.

20. Fleetwood K, McCool R, Glanville J, Edwards SC, Gsteiger S, Daigl M, et al. Systematic review and network meta-analysis of idiopathic pulmonary fibrosis treatments. J Manag Care Spec Pharm. 2017;23(3-b):S5.

21. Loveman E, Copley VR, Colquitt JL, Scott DA, Clegg AJ, Jones $\mathrm{J}$, et al. The effectiveness and cost-effectiveness of treatments for idiopathic pulmonary fibrosis: systematic review, network metaanalysis and health economic evaluation. BMC Pharmacol Toxicol. 2014;15(63): 13 .

22. Rochwerg B, Neupane B, Zhang Y, Garcia CC, Raghu G, Richeldi $\mathrm{L}$, et al. Treatment of idiopathic pulmonary fibrosis: a network meta-analysis. BMC Med. 2016;14:18.

23. Skandamis A, Kani C, Markantonis SL, Souliotis K. Systematic review and network meta-analysis of approved medicines for the treatment of idiopathic pulmonary fibrosis. J Drug Assess. 2019;8(1):55-61.

24. Noble PW, Albera C, Bradford WZ, Costabel U, Glassberg MK, Kardatzke D, et al. Pirfenidone in patients with idiopathic pulmonary fibrosis (CAPACITY): two randomised trials. Lancet. 2011;377(9779):1760-9.

25. Kondoh Y, Taniguchi H, Katsuta T, Kataoka K, Kimura T, Nishiyama $\mathrm{O}$, et al. Risk factors of acute exacerbation of idiopathic pulmonary fibrosis. Sarcoidosis Vasc Diffuse Lung Dis. 2010;27(2):103-10.

26. Boehringer Ingelheim. Summary of clinical safety: nintedanib (BIBF 1120) (data on file). Ingelheim am Rhein: Boehringer Ingelheim; 2014.
27. Boehringer Ingelheim. Highlights of prescribing information. OFEV (nintedanib) capsules for oral use. Ingelheim am Rhein: Boehringer Ingelheim; 2014.

28. Dolan P. Modeling valuations for EuroQol health states. Med Care. 1997;35(11):1095-108.

29. Ara R, Brazier JE. Using health state utility values from the general population to approximate baselines in decision analytic models when condition-specific data are not available. Value Health. 2011;14(4):539-45.

30. Institut national d'assurance maladie-invalidité (INAMI). Online database. Available from: https://www.tctfgovbe/webetct/etctweb/. Accessed 1 Jan 2019.

31. Electronic Medicines Compendium (eMC). Esbriet $267 \mathrm{mg}$ hard capsules: Summary of product characteristics (SPC). Available from: http://www.medicinesorguk/emc/medicine/29932. Accessed May 2014.

32. Electronic Medicines Compendium (eMC). Ofev $100 \mathrm{mg}$ and $150 \mathrm{mg}$ soft capsules: Summary of product characteristics (SPC). Available from: http://www.medicinesorguk/emc/medicine/30110 . Accessed May 2014.

33. Simoens S, Kutten B, Keirse E, Vanden Berghe P, Beguin C, Desmedt $\mathrm{M}$, et al. Terminal patients in Belgian nursing homes: a cost analysis. Eur J Health Econ. 2013;14(3):407-13.

34. Neyt M, Huybrechts M, Hulstaert F, Vrijens F, Ramaekers D. Trastuzumab in early stage breast cancer: a cost-effectiveness analysis for Belgium. Health Policy. 2008;87(2):146-59.

35. Fust K, Li X, Maschio M, Villa G, Parthan A, Barron R, et al. Cost-effectiveness analysis of prophylaxis treatment strategies to reduce the incidence of febrile neutropenia in patients with earlystage breast cancer or non-Hodgkin lymphoma. PharmacoEconomics. 2017;35(4):425-38.

36. Husereau D, Drummond M, Petrou S, Carswell C, Moher D, Greenberg D, et al. Consolidated Health Economic Evaluation Reporting Standards (CHEERS) statement. Br Med J (Clin Res Ed). 2013;346:f1049.

37. Canestaro WJ, Forrester SH, Raghu G, Ho L, Devine BE. Drug treatment of idiopathic pulmonary fibrosis: systematic review and network meta-analysis. Chest. 2016;149(3):756-66.

38. Yu YF, Macauley DS, Reichmann WM, Wu EQ, Nathan SD. Association of early suspected acute exacerbations of idiopathic pulmonary fibrosis with subsequent clinical outcomes and healthcare resource utilization. Respir Med. 2015;109(12):1582-8. 